\title{
A Survey on Color Image Enhancement Techniques
}

\author{
Adlin Sharo T ${ }^{1}$, Kumudha Raimond ${ }^{2}$ \\ ${ }^{1}$ (Computer Science and Engineering, Karunya University, India) \\ ${ }^{2}$ (Computer Science and Engineering, Karunya University, India)
}

\begin{abstract}
Improving visual clarity of an image is conveniently achieved by various contrast enhancement techniques. Histogram equalization results in excess contrast enhancement. When fuzzy concepts are used for color image enhancement it creates visual artifacts. But contrast enhancement using ACO (Ant Colony Optimization) technique avoids excess contrast enhancement and visual artifacts are discussed in this paper.
\end{abstract}

Keywords: Ant colony optimization, Fuzzy logic, Histogram equalization, HSV, Image enhancement.

\section{INTRODUCTION}

The process of enhancing the visual data in an image is termed as Image enhancement. This process helps to maximize the clarity of the captured image. Because of the limited capabilities of the hardware device which is used for image acquisition the atmospheric effects such as mist, fog and cloud adds unwanted information results the image to blur. In addition to this image enhancement technique is needed in many areas such as remote sensing, robot navigation, textiles, military, film industry, document processing, graphic arts, printing industry, biomedical image analysis and forensic image analysis. Several techniques have been developed to serve this purpose. Our goal in this paper is to have an analysis of various image enhancement technique helps to implement contrast enhancement.

\section{CONTRAST ENHANCEMENT TECHNIQUES FOR IMAGES \\ 2.1 Histogram Technique}

Histogram equalization is a technique for adjusting image intensities to enhance contrast. In histogram equalization the pixel will be uniformly distributed instead of original pixel distribution and hence the image gains more clarity.

Duan have introduced a novel histogram processing algorithm which considers the original distribution of pixel in the equalization process [1]. The luminance and saturation component of pixel is changed and the hue component remains constant. The development of the algorithm shows more contrast and the image looks natural and avoid visual artifacts. Author T. Arich have introduced a low-complexity algorithm for contrast enhancement [2]. Histogram Modification (HM) techniques such as Adjustable Histogram Equalization , Histogram Smoothing, Weighted Histogram Approximation, Black and White Stretching is done by computing histogram and by adjusting the level of enhancement. Hence, the contrast of the image can be improved without introducing visual artifacts, but the enhanced image is not visually more pleasing. The original image and its histogram is shown in Fig 3.3.

\subsection{Fuzzy Logic Approach}

Fuzzy means vagueness, Fuzziness occurs when the boundary of a piece of information is not clear. M.Hellmann introduced classical set theory to mention the elements using the set $\mathrm{B}=\{\mathrm{b} 1, \mathrm{~b} 2, \mathrm{~b} 3, \ldots \ldots \mathrm{bn}\}$. If the elements present in set ' $B$ ' are subset of universal set ' $X$ ' then set $B$ can be represented as $x \varepsilon$ X by its characteristic function. A fuzzy is represented by a membership function $\mu \mathrm{B}(\mathrm{x})$ of $\mathrm{B}$. Operations performed on fuzzy sets are AND, OR, NEGATION. Fuzzy classification is mainly done by "IF...THEN...ELSE" and rule based smoothing, then it is defuzzified to get crisp output. Fuzzy Logic method is used to solve classification problem [3].

To remove noise from an image and to enhance it, B. Tang et.al introduced a new approach [12]. This algorithm is based on separating the color data into chromaticity and brightness, and then processing each one of these components with partial differential equations or diffusion flows. Under exposed and overexposed images are not properly enhanced. In the digital image processing field to enhance an image, the noise present is removed by using filter. Manglesh Khandelwal proposed a new algorithm to enhance color image corrupted by Gaussian noise using fuzzy logic. The fuzzy logic rules are generated. Then the RGB values of image is passed to fuzzy logic approach. Fuzzy output will differentiate between ambiguous colors [4]. Sarode M.K.V have introduced a new algorithm to retrieve the features related to a specific tumor disease. Hue is preserved, only saturation and value is changed and then fuzzy logic rules are applied, based on this decisions related to 
classification of colors will be done. The output will be consisting of only the serious tumor region and noisy pixels was removed [5].

Fuzzy intensification method was proposed by M. Hanmandlu in which a new contrast intensification operator is introduced, which consist of parameter ' $\mathrm{t}$ '. By preserving $\mathrm{H}$, and changing only $\mathrm{S}$ and $\mathrm{V}$ and by minimizing the fuzzy entropy, the quality of image is visually improved for human contrast perception [6]. In order to maintain human visual perception Lior Shamir proposed a human perception based approach to pixel color segmentation [16]. Fuzzy sets are classified based on the H, S and V color components. Hue is defined using Red, Dark Orange, Light Orange, Yellow, Light Green, Dark Green, Aqua, Blue, Dark Purple, Light Purple. Saturation is defined using the five fuzzy sets Gray, Almost Gray, Medium, Almost Clear, Clear. Value is defined using the four fuzzy sets Dark, Medium Dark, Medium Bright and Bright. The fuzzy rules in this model are defined based on human observations. For example, the rule "Dark Orange, Medium, Medium Dark gives Dark Brown" is defined manually by classifying the HSV color component. Fuzzy logic-based method provides a more accurate color classification.

In [11], author H.D. Cheng proposed a novel adaptive direct fuzzy contrast enhancement method, sigmoidal membership function is used to map an image from spatial to fuzzy domain. The resultant image obtained is properly enhanced. While transforming the image from one color space (RGB) to another color space (HSV, HIS, YIQ) hue is unaltered only the intensity and saturation components are changed, as a result gamut problem will occur. So in [14], S.K.Naik tried to keep the transformed values within the range of the RGB space so as to avoid the gamut problem. Even though the image is enhanced, the clarity of the enhanced image is not good.

A HVS controlled Color Image Enhancement and Evaluation algorithm (HCCIEE algorithm) proposed by K.-Q. Huang which mostly concentrate on the visual effect and the image is enhanced without ringing or halo artifacts [13]. By using various algorithms the degraded images are enhanced, but sometimes the image may be over enhanced. So in order to rectify this problem, in [10] the D. Yu have introduced hue preserving correction algorithm named normalized SI correction appropriate for different kind of enhancement. The visual quality of the image is enhanced but the process is time consuming. The comparison with fuzzy algorithms is shown in TABLE 3.1 and the comparison of algorithms other than fuzzy is shown in TABLE 3.2.

\subsection{Optimization Technique}

In order to enhance the image many optimization techniques are used such as greedy, genetic, bacterial foraging, ant colony system. Madasu Hanmandlu introduced a Guassian membership function to fuzzify the image which consist of intensification parameter $t$, fuzzifier fh, and the crossover point for enhancement of color images. By minimizing the fuzzy entropy of the image the parameters are optimized, thus a visible image quality is observed for under exposed images [7]. John See proposed a novel fuzzy-based Gaussian edge detector uses both global and local image properties. In the global contrast intensification phase, Gaussian membership function is used. A nonlinear contrast intensification function consist of three parameters is used to enhance the image. In the local phase, Gaussian-type edge detector mask containing two fuzzifier parameters is optimized by using fuzzy image entropy function and this algorithm showed notable visual improvement. S.Palanikumar have introduced a new algorithm based on HE and Adaptively Increasing Value Histogram Equalization (AIVHE) [9]. By using AIVHE method, the contrast is enhanced and is controlled by two user parameters beta and gamma, but the detailed information in the palmprint is not preserved. In order to further enhance the palmprint and to preserve the information, Genetic Algorithm (GA) is used to optimize the value of beta and gamma by minimizing entropy value.

A greedy heuristic approach, controlled by a single parameter, to approximate the optimization problem which includes a perceptual constraint derived from human supra threshold contrast sensitivity function which follows weber law is proposed by Aditi Majumder. This method is free of artifacts, but it is not applicable in case of video [15].

Image consist of many clustered object that overlap each other and hid the structure which lead to the problem of structure extraction. In order to solve classification problem Subba Rao Katteda et.al have proposed an algorithm called Ant Colony Optimization and Fuzzy logic based technique [17]. By using fuzzy logic rules are formed and by using ant colony optimization each pixel intensity value is collected separately. Based on the fuzzy logic rules the pixels are grouped together to retrieve the structure, but it is very complex to form fuzzy rules.

A Novel Fuzzy Ant Colony System for parameter determinations proposed by C.W.Tao which is based on the fuzzy rules, the transition behavior of ants is initiated. The transition probability rules are newly created to choose the next city. Thus the proper parameters of the fuzzy sliding controllers for swinging and balancing the inverted pendulum and cart system is found [18]. Vittorio Maniezzo in his work he has introduced a metaheuristic algorithm for combinatorial optimization problems. Ant System (AS) was initially applied to the solution of the traveling salesman problem, then ACO is applied to find better solution [19]. In AS the 
pheromone is updated for all the solutions obtained by ant colony. In ACS all ants are iterated and at the end of each iteration, pheromone is updated only for the best solution. So the ant find the route easily and it avoids long convergence time.

Ant Colony Optimization (ACO) is an optimization technique inspired by the behavior of real ant, how ants deposit pheromone for finding food. J. Tian, have introduced ACO to solve the edge detection problem. ACO based edge detection approach develops a pheromone matrix which shows the edge information on each pixel position. Construction process and the update process are done iteratively to develop the pheromone matrix. Finally, the edge is determined by using the decision process, but the computational load need to be reduced [20]. S. Meshoul have proposed to achieve robust point matching and pose estimation for image registration purpose from noisy and outlying information. Ant Colony System (ACS) is used as a search strategy to identify the initial solutions. A local search is included along within the search strategy to refine the solution obtained. The convergence is achieved and thus the accuracy is improved, but it can be stable up to $30 \%$ of outlier data [21].

Several difficult optimization problems are solved by algorithms which is attracted by the foraging behavior of real ant species. M. Dorigo has defined Ant Colony Optimization (ACO) meta-heuristic by applying first to solve the problem of travelling salesman problem to find minimal length Hamiltonian circuit on the graph. Then in the network communication by using antnet ACO is used to search for a minimum cost path between a pair of nodes [22]. R.S. Parpinelli have proposed an algorithm for data mining called Ant-Miner The objective of this algorithm is to retrieve classification rules from data. The algorithm is based on the behavior of real ant colonies and some data mining techniques [23]. The rules produced by Ant-Miner is less compared to $\mathrm{CN} 2$, which is a data mining algorithm for classification of data sets based on threshold. The results obtained by Ant-Miner algorithm shows more accuracy than CN2.

An image can be grouped into underexposed, mixedexposed and overexposed regions based on the exposure. The RGB image is converted into HSV image. The hue is preserved only the luminance component of the image is changed. Fuzzification is done on the under and over exposed region by using Guassian membership function based on crossover point and the intensification parameter. The optimum values of these parameters are obtained by the modified AACS [24] optimization technique iteratively. The enhanced image produced by this approach is visually more pleasing.

\section{Figures and Tables}

Table 3.1 Comparison with fuzzy algorithms

\begin{tabular}{|c|c|c|c|c|c|c|}
\hline Reference & $\begin{array}{l}\text { Color } \\
\text { space }\end{array}$ & $\begin{array}{l}\text { HSV } \\
\text { used }\end{array}$ & Entropy & $\begin{array}{c}\text { Type of } \\
\text { Membership } \\
\text { function }\end{array}$ & $\begin{array}{l}\text { Optimization } \\
\text { Technique }\end{array}$ & Optimization parameter \\
\hline Om Prakash Vermaa, et.al., 2011, [24] & HSV & $\mathrm{S}, \mathrm{V}$ & $\mathrm{E}$ & $\begin{array}{l}\text { Guassian, } \\
\text { Sigmoidal }\end{array}$ & $\mathrm{ACO}$ & $\begin{array}{l}\text { Intensification factor, } \\
\text { pivot }\end{array}$ \\
\hline M.Hellman, et.al, 2000, [3] & Grey & - & - & Triangle & - & - \\
\hline Manglesh Khandelwal, et.al, 2005, [4] & HSV & $\mathrm{H}, \mathrm{S}, \mathrm{V}$ & - & Trapezoidal & - & - \\
\hline Sarode M.K.V., et.al, 2008, [5] & HSV & $\mathrm{S}, \mathrm{V}$ & - & Trapezoidal & - & - \\
\hline M.Hanmandl, et.al, 2003, [6] & HSV & $\mathrm{S}, \mathrm{V}$ & $\mathrm{E}$ & $\begin{array}{l}\text { Guassian, } \\
\text { sigmoidal }\end{array}$ & - & - \\
\hline Madasu Hanmandlu, et.al, 2006, [7] & HSV & $\mathrm{V}$ & $\mathrm{E}$ & $\begin{array}{l}\text { Guassian, } \\
\text { sigmoidal }\end{array}$ & $\begin{array}{l}\text { Modified } \\
\text { univariate } \\
\text { algorithm }\end{array}$ & $\begin{array}{l}\text { Intensification parameter, } \\
\text { crossover point, fuzzifier }\end{array}$ \\
\hline John See, et.al, 2005, [8] & Grey & - & $\mathrm{E}$ & $\begin{array}{l}\text { Guassian, } \\
\text { sigmoidal }\end{array}$ & Entropy & $\begin{array}{c}\text { Fuzzifier, crossover } \\
\text { point, adjustable fuzzifier }\end{array}$ \\
\hline H.D. Cheng, et.al, 2000,[11] & Grey & - & $\mathrm{E}$ & $\begin{array}{l}\text { Sigmoidal } \\
\text { function }\end{array}$ & - & - \\
\hline Lior Shamir, et.al, 2006,[16] & HSV & $\mathrm{H}, \mathrm{S}, \mathrm{V}$ & - & Triangle & - & - \\
\hline Subba Rao Katteda, et.al, 2011, [17] & Grey & & - & Trapezoidal & $\mathrm{ACO}$ & Intensity value \\
\hline C.-W. Tao, et.al, 2009,[18] & - & - & - & Trapezoidal & $\mathrm{ACO}$ & Fuzzy controller \\
\hline
\end{tabular}


Table 3.2 Comparison with algorithms other than fuzzy

\begin{tabular}{|c|c|c|c|c|c|}
\hline Reference & Color space & HSV used & Algorithm & $\begin{array}{c}\text { Optimization } \\
\text { Technique }\end{array}$ & $\begin{array}{c}\text { Optimization } \\
\text { parameter }\end{array}$ \\
\hline J. Duan, 2004, [1] & Grey, HSV & $\mathrm{S}, \mathrm{V}$ & HM & - & - \\
\hline T. Arich, 2009, [2] & Grey, RGB & - & $\mathrm{HM}$ & - & - \\
\hline S.Palanikumar, 2012, [9] & Grey & - & GA and $\mathrm{HE}$ & Genetic & $\begin{array}{l}\text { User controlled } \\
\text { parameter }\end{array}$ \\
\hline D. Yu, 2007, [10] & HSV & $\mathrm{S}, \mathrm{V}$ & $\begin{array}{l}\text { Normalized SI } \\
\text { correction }\end{array}$ & - & - \\
\hline B. Tang, 2001, [12] & HSV & $\mathrm{S}, \mathrm{V}$ & $\begin{array}{c}\text { Brightness, } \\
\text { chromaticity diffusion }\end{array}$ & - & - \\
\hline K.-Q. Huang, 2007, [13] & HSV & - & HCCIEE & - & - \\
\hline S.K. Naik, 2003, [14] & HSV & $\mathrm{S}, \mathrm{V}$ & Transformation, HE & - & - \\
\hline Aditi Majumder, 2006, [15] & Grey, HSV & $\mathrm{S}, \mathrm{V}$ & Greedy iterative & Greedy & Scalar functions \\
\hline
\end{tabular}
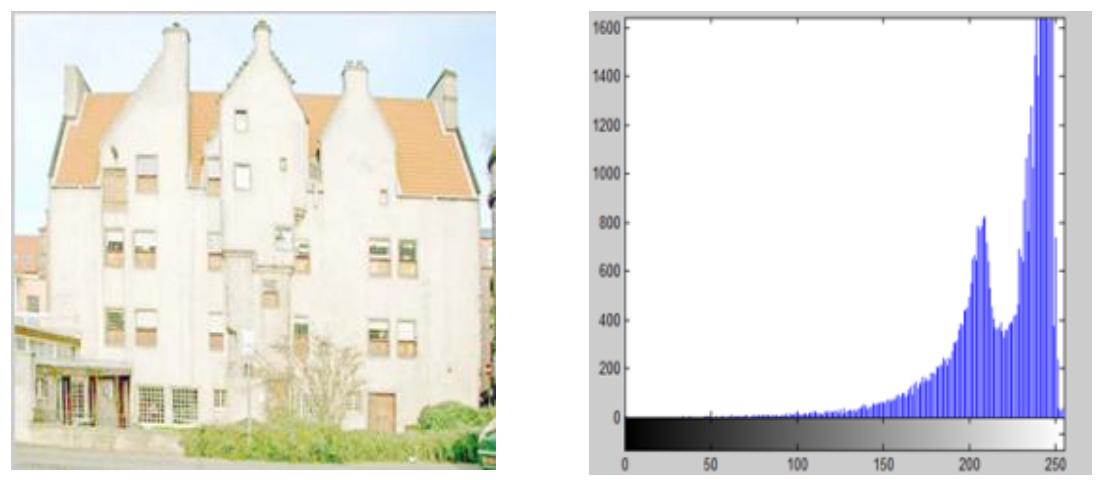

Fig 3.3 Original image and its histogram.

\section{Conclusion}

In this paper, a survey on various techniques for color image enhancement is carried out with the existing methods like histogram equalization, fuzzy based methods and other optimization techniques those used for image contrast enhancement. Histogram equalization method leads to excess enhancement in-order to overcome it fuzzy is used. Fuzzy rule based technique and its computation gives very ample improvement in contrast enhancement whereas better results are obtained when optimization techniques like ACO is used. By using other bio-inspired optimization techniques may give quick computation process for image enhancement.

\section{References}

[1] J. Duan, G. Qiu, "Novel histogram processing for colour image enhancement", Third International Conference on Image and Graphics, 4, 2004, 55-58.

[2] T. Arich, S. Dikbas, "A histogram modification framework and its application for image contrast enhancement", IEEE Trans. Image Process. , 18, 2009.

[3] M.Hellmann, "Fuzzy logic Introduction", Info. \& Ctl., 12, 1968, 94-102.

[4] Manglesh Khandelwal, Shweta Saxena, Priya Bharti, "An efficient algorithm for Image Enhancement", Indian Journal of Computer Science and Engineering (IJCSE), 2, 2005, 118-123.

[5] Sarode M.K.V, S.A. Ladhake, P.R. Deshmukh "Fuzzy system for color image enhancement" world academy of science, Engineering \& Technology, 48, 2008, 311-316.

[6] M. Hanmandlu, D. Jha, R. Sharma, "Color image enhancement by fuzzy intensification", Pattern Recognition. Lett., 24, 2003, 81-87.

[7] Madasu Hanmandlu,Member, IEEE and Devendra Jha, “An optimal Fuzzy System for color image enhancement", IEEE Trans., 15, 2006, 2956-2966. 
[8] John See, Madasu Hanmandlu, Shantaram Vasikarla, "Fuzzy based Parameterized Guassian Edge Detector Using Global and Local Properties", International Conference on Information Technology, 02, 2005, $101-106$.

[9] S. Palanikumar, M. Sasikumar, J. Rajeesh, "Entropy Optimized Palmprint Enhancement Using Genetic Algorithm and Histogram Equalization”, International Journal of Genetic Engineering, 2, 2012, 12 18.

[10] D. Yu, L.-H. Ma, H.-Q. Lu, "Normalized SI correction for hue-preserving color image enhancement", International Conference on Machine Learning and Cybernetics, 2007, pp. 1498-1503.

[11] H.D. Cheng, Huijuan $\mathrm{Xu}$, "A novel fuzzy logic approach to contrast enhancement", Pattern Recognition, 33 , 2000, 809-819.

[12] B. Tang, G. Sapiro, V. Caselles, "Color image enhancement via chromaticity diffusion", IEEE Trans. Image Process., 10 , 2001, 701-707.

[13] K.-Q. Huang, Q. Wang, Z.-Y. Wu, "Natural color image enhancement and evaluation algorithm based on human visual system", Comput. Vis. Image Underst., 103, 2006, 52-63.

[14] S.K. Naik, C.A. Murthy, "Hue-preserving color image enhancement without gamut problem", IEEE Trans. Image Process., 12, 2003, 1591-1598.

[15] Aditi Majumder, Sandy Irani, "Contrast Enhancement of Images using Human Contrast Sensitivity”, Applied perception in graphics and visualization, 11, 2006, 69-76.

[16] Lior Shamir, "Human Perception-based Color Segmentation Using Fuzzy Logic", IPCV, 2, 2006, 96502 .

[17] Subba Rao Katteda , Dr.C Naga Raju, and Maddala Lakshmi Bai, "Feature Extraction for Image Classification and Analysis with Ant Colony Optimization Using Fuzzy Logic Approach", Signal and image processing, An International Journal (SIPIJ), 2(4), 2011.

[18] C.-W. Tao, J.-S. Taur, J.-T. Jeng, and W.-Y. Wang, “A Novel Fuzzy Ant Colony System for Parameter Determination of Fuzzy Controllers", International Journal of Fuzzy Systems, 11(4), 2009.

[19] Vittorio Maniezzo, Luca Maria Gambardella, Fabio de Luigi, "Ant Colony Optimization, Optimization Techniques in Engineering”. Springer-Verlag conference, 141,2004, 101-117.

[20] J. Tian, W. Yu, S. Xie, "An ant colony optimization algorithm for image edge detection", IEEE Congress on Evolutionary Computation, 9, 2008, 751-756.

[21] S. Meshoul, M. Batouche, "Ant colony system with extremal dynamics for point matching and pose estimation", International Conference on Pattern Recognition, 3, 2002, 823-826.

[22] M. Dorigo, G.D. Caro, "Ant colony optimization: a new meta-heuristic", Proceedings of the Congress on Evolutionary Computation, IEEE, 2, 1999, 1470-1477.

[23] R.S. Parpinelli, H.S. Lopes, A.A. Freitas, "Data mining with an ant colony optimization Algorithm", IEEE Trans. Evol. Comput., 6, 2002, 321-332.

[24] Om Prakash Vermaa, Puneet Kumarb, Madasu Hanmandluc, Sidharth Chhabrad, "High dynamic range optimal fuzzy color image enhancement using Artificial Ant Colony System”, Applied Soft Computing, 12, 2011, 394-404. 